\title{
eIMMR: the future of health statistics in Sri Lanka
}

\author{
Dr. N.C. Kariyawasam
}

Post graduate Institute of Medicine, University of Colombo

Dr. V.S. Weerasekera

Post graduate Institute of Medicine, University of Colombo

Dr. M.K.D.R.B. Dayaratne

Post graduate Institute of Medicine, University of Colombo

\section{Dr. R. Hewapathirana}

Post graduate Institute of Medicine, University of Colombo

\section{Dr. R.P.P. Karunapema}

Non communicable diseases Unit, Ministry of Health

\section{Dr. I.R. Bandara}

Medical Statistics Unit, Ministry of Health

Contact e-mail address: overedu@sltnet.lk

eHealth Sri Lanka 2010,1(suppl.1):S14

DOI: http://dx.doi.org/10.4038/sljbmi.v1i0.3549

Only the Abstract is available

\begin{abstract}
Indoor Morbidity and Mortality Record (IMMR) forms the back bone of data in the evaluation of the health status of a nation. In Sri Lanka it is also an integral part of the health information system which supports management of healthcare, monitoring of disease patterns and evaluation of treatment and prevention. Although the paper based system of IMMR in place has served this purpose over the years to a level of satisfaction, it has increasingly become obsolete with practical and logistical barriers. This has rendered it almost a daunting task to keep up with the expected levels of timeliness, accuracy and relevancy as is required. It has become ever more evident in the recent past with the volatility of disease patterns on the rise and the role of disease surveillance becoming a "life-saver" in current epidemics. In the quest for solutions, healthcare digitisation along with a web-based IMMR, as explored in this instance, proved to be a promising tool and a shift in the right direction. The system introduced will pave the way for the concept of "enter once-use many times" of all data inputs plus the almost unimaginable ways of analysis and presentations. With almost real-time information at hand and extrapolations of such, the digital healthcare information system would inevitably become the "crystal ball" for prediction of the future health needs and disease patterns.
\end{abstract}

Keywords - IMMR, volatility of disease patterns, digital healthcare information system 
